Check for updates

Cite this: RSC Adv., 2020, 10, 31765

\title{
Biophotonic sensor for the detection of creatinine concentration in blood serum based on 1D photonic crystal
}

\author{
Arafa H. Aly, (D) *a Doaa Mohamed, ${ }^{a}$ Mona A. Mohaseb, ${ }^{\text {ab }}$ N. S. Abd El-Gawaad ${ }^{c}$ \\ and $Y$. Trabelsi ${ }^{\text {de }}$
}

A new biophotonic sensor based on photonic crystal (PC) has been designed for the detection of creatinine concentration in blood, and is considered an important small molecule biomarker of renal dysfunction. Based on the transfer matrix method (TMM), we theoretically investigated the transmittance spectra of a one dimensional alternating dielectric photonic crystal (PC) designed as $(A B)^{7} / C /(A B)^{7}$ made of $M F_{2}$ (A), $\mathrm{CeO}_{2}$ (B) and creatinine concentration present in blood (C). The transmission spectra exhibit resonant peaks within the photonic band gap (PBG) indicative of so-called defect modes, which depend on parameters, such as concentration of creatinine in blood, thickness of defect layer and incident angle. The proposed sensor can determine the physiological levels of creatinine in human blood serum samples. The estimated parameters realize an efficient biophotonic sensor wherein sensitivity was tuned from $136.4 \mathrm{~nm}$ per RIU to $306.25 \mathrm{~nm}$ per RIU and is very useful for the detection of creatinine.

Received 21st June 2020

Accepted 29th July 2020

DOI: $10.1039 / \mathrm{dOra05448h}$

rsc.li/rsc-advances

Amongst the sensors, biosensors are devices with mecha-

\section{Introduction}

Photonic crystals are periodic refractive index structural arrays as a function of one-dimension, two-dimension, or all threedimension space. At the interface between every two different layers, a portion of the incident wave is reflected and due to a destructive interference between the incident wave and reflected wave, a standing wave is formed.

Photonic crystals (PCs) are structures of nanometric compositions of insulating and metal-insulating materials discovered by Yablonovitch in 1987. ${ }^{1}$ These structures present a modulation of refractive indices that can control the propagation of electromagnetic waves and is called the photonic band gap (PBG). ${ }^{2}$ The PBG consists of a range of frequencies that forbid the propagation of light and are highly sensitive to external conditions, including mechanical stress, ${ }^{3}$ temperature,${ }^{4,5}$ electric field, ${ }^{6}$ magnetic field, ${ }^{7}$ chemicals, ${ }^{8}$ pressure, ${ }^{9}$ and self-organization of multilayered stacks. ${ }^{10,11}$ Therefore, PCs are considered the basis for sensing applications, such as a chemical sensors, ${ }^{12}$ pressure sensors, ${ }^{13}$ and biosensors. ${ }^{14}$

${ }^{a}$ TH-PPM Group, Physics Department, Beni-Suef University, Beni Suef, Egypt. E-mail: arafa.hussien@science.bsu.edu.eg

${ }^{b}$ Department of Physics, Faculty of Applied Science, Umm-Al-Qura University, Mecca, Saudi Arabia

'Faculty of Science, King Khalid University, Abha, Saudi Arabia

${ }^{d}$ Physics Department, College of Arts and Sciences in Muhail Asir, King Khalid University, Abha, Saudi Arabia

${ }^{e}$ Photovoltaic and Semiconductor Materials Laboratory, National Engineering School of Tunis, University of Tunis El Manar, 1002, Tunis, Tunisia nisms for measuring changes in biological systems. Researchers have used different sensors to detect the shift in hormones, ${ }^{15}$ enzymes,${ }^{16}$ level of glucose in urine, ${ }^{17}$ cancerolic cells, ${ }^{18}$ and nucleic acids. ${ }^{19}$ Many new technologies use materials, such as hydrogels, nanoparticles and PCs for developing compact biosensing systems. ${ }^{20-25}$ Among them, PC-based biosensor technology is considered a simple and cost-effective method to observe various diseases much more efficiently than traditional methods.

Therefore, the considered PC technology is adequate to sense the complexity of structures of biological molecules as well as overcome the high manufacturing cost of sensors. ${ }^{26-29}$ Furthermore, PC-based biosensors are widely used as a diagnostic tool for assessing kidney dysfunction by measuring creatinine concentration, ${ }^{30}$ glucose $\mathrm{e}^{31-33}$ and cholesterol $^{34}$ in body fluids. There are many types of biosensors that convert signals from biological systems to electrochemical, optical, electrical or magnetic signals.

Optical sensors, in particular, play an important role in the medical field because they provide vital and complementary information. ${ }^{35}$ The metabolic processes in biological systems are accompanied by chemical wastes called creatinine. The creatinine is mostly responsible for the energy production in muscles. Hence, disposal of creatinine from the biological system is extremely crucial.

This operation is done by the transfer of creatinine through the blood to the kidney, which disposes it through urine. Further, the measurement of levels of creatinine in blood is considered as a strong benchmark for efficient kidney 
functioning. ${ }^{36,37}$ Therefore, the process of measuring the levels of creatinine in the blood is a very important step to identify kidney diseases. ${ }^{38,39}$ There are many methods to determine creatinine levels in blood and urine, such as Jaffé's method ${ }^{\mathbf{4 0}}$ as well as enzymatic methods. ${ }^{\mathbf{4 1 , 4 2}}$

In this study, we present a new type of biosensor based on 1D PCs that is able to estimate the levels of creatinine in blood by taking into account device factors, such as convenience, costefficiency of the equipment, and optimizing the time gap. Based on the TMM approach, we theoretically studied the transmission spectrum of the PC containing a creatinine sample under different concentrations. We estimated the related parameters in order to improve the sensitivity of the considered photonic biosensor.

\section{Theoretical model}

We theoretically studied the transmittance spectra of a $1 \mathrm{D}$ defective PC designed as $(\mathrm{AB})^{m} / C /(\mathrm{AB})^{m}$ made up of $\mathrm{MgF}_{2}(\mathrm{~A})$, $\mathrm{CeO}_{2}(\mathrm{~B})$ and creatinine concentration in blood $(C)$, where $m$ is the number of periods. The configuration of the proposed $1 \mathrm{D}$ multilayered stack is shown in Fig. 1.

Based on the transfer matrix method (TMM), the interaction between stratified medium and the electromagnetic waves is described by the following equation: ${ }^{\mathbf{4 3 , 4 4}}$

$$
H=\left(\begin{array}{ll}
H_{11} & H_{12} \\
H_{21} & H_{22}
\end{array}\right)=\left(h_{1} h_{2}\right)^{m} h_{\mathrm{d}}\left(h_{1} h_{2}\right)^{m}
$$

where $\left(H_{i i}\right)_{i=1,2}$ are the elements of the transfer matrix. Further, $h_{1}, h_{2}, h_{\mathrm{d}}$ are the characteristic matrix of stratified layers: $\mathrm{MgF}_{2}$, $\mathrm{CeO}_{2}$ and creatinine, respectively, and can be defined as following: ${ }^{43,44}$

$$
h_{\mathrm{r}}=\left(\begin{array}{cc}
\cos \theta_{\mathrm{r}} & \frac{-i \sin \theta_{\mathrm{r}}}{p_{\mathrm{r}}} \\
-i p_{\mathrm{r}} \sin \theta_{\mathrm{r}} & \cos \theta_{\mathrm{r}}
\end{array}\right)
$$

with $\theta_{\mathrm{r}}=\frac{2 \pi n_{\mathrm{r}} d_{\mathrm{r}} \cos \psi_{\mathrm{r}}}{\lambda}$ as the phase difference at each interface. However, $\hat{p}_{\mathrm{r}}$ denotes the optical thicknesses for the $\mathrm{S}$ polarized wave that satisfies the equation: $P_{\mathrm{r}}=n_{\mathrm{r}} \cos \psi_{\mathrm{r}}$, where $\psi_{1}, \psi_{2}, \psi_{\mathrm{d}}$ are the incident angles of corresponding materials: $\mathrm{MgF}_{2}, \mathrm{CeO}_{2}$ and creatinine, respectively, satisfying the Snell's law:

$$
n_{\mathrm{o}} \sin \psi_{\mathrm{o}}=n_{1} \sin \psi_{1}=n_{2} \sin \psi_{2}=n_{\mathrm{d}} \sin \psi_{\mathrm{d}}
$$

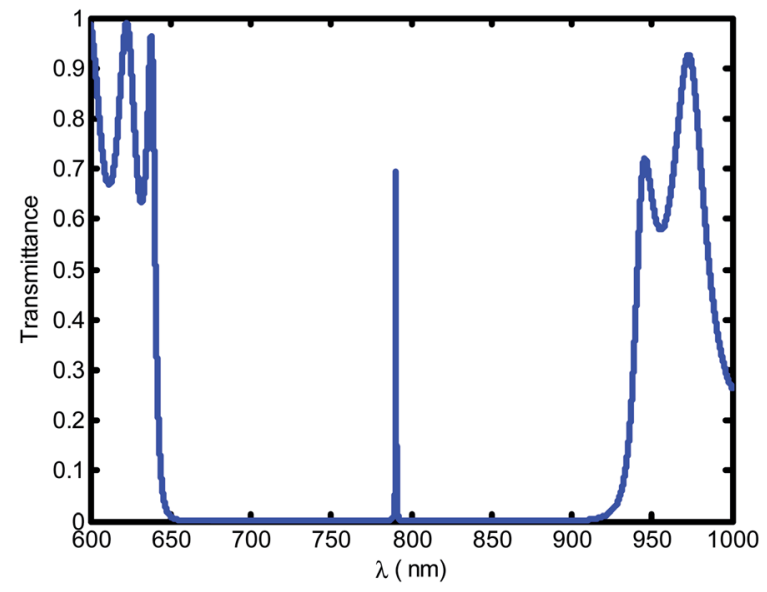

Fig. 2 The transmittance spectrum of a defective photonic crystal as a function of wavelength at blood creatinine sample $\left(80.9 \mu \mathrm{mol} \mathrm{L}{ }^{-1}\right)$.

Table 1 Creatinine concentration $\left(\mu \mathrm{mol} \mathrm{L}{ }^{-1}\right)$ with attributed refractive index

\begin{tabular}{ll}
$\begin{array}{l}\text { Refractive } \\
\text { index }\end{array}$ & $\begin{array}{l}\text { Creatinine concentration } \\
\left.(\mu \mathrm{mol} \mathrm{L})^{-1}\right)\end{array}$ \\
\hline 2.661 & 80.9 \\
2.655 & 81.43 \\
2.639 & 82.3 \\
2.610 & 83.3 \\
2.589 & 84.07 \\
2.565 & 85.28
\end{tabular}

The $2^{\text {nd }}$ kind of Chebyshev polynomials are used to calculate the matrices product $\left(h_{1} h_{2}\right)^{m}$. $^{44}$

Consequently, the transmittance satisfies the following expression: ${ }^{45}$

$$
t=\frac{2 P_{o}}{H_{11} H_{12} P_{0}+\left(H_{21}+H_{21} H_{22} P_{\mathrm{s}}\right)}
$$

where $P_{\mathrm{o}}$ and $P_{\mathrm{s}}$ are the values of transmittance of air and substrate, respectively, and can be determined by the elements of the transfer matrix.

For the case of TE mode, the transmittance is given by the following expression: ${ }^{\mathbf{4 4}}$

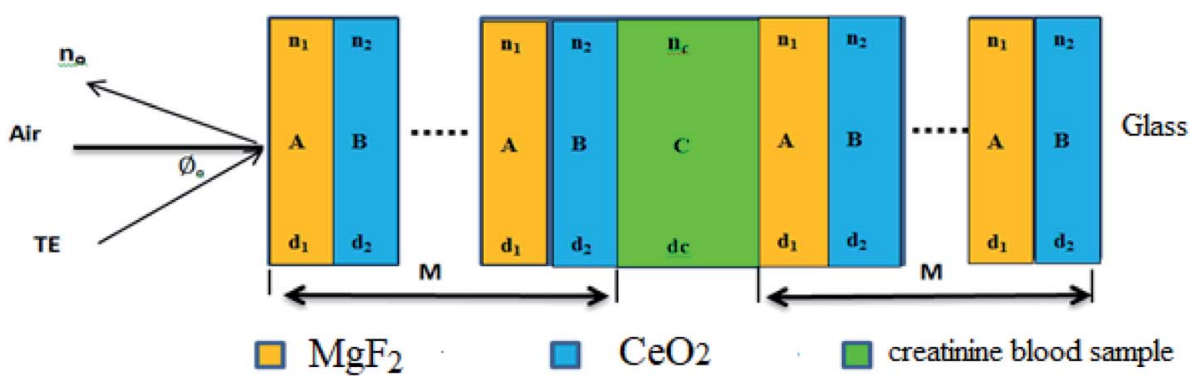

Fig. 1 Schematic of the proposed biosensor based on 1D defective photonic crystal (PC). 


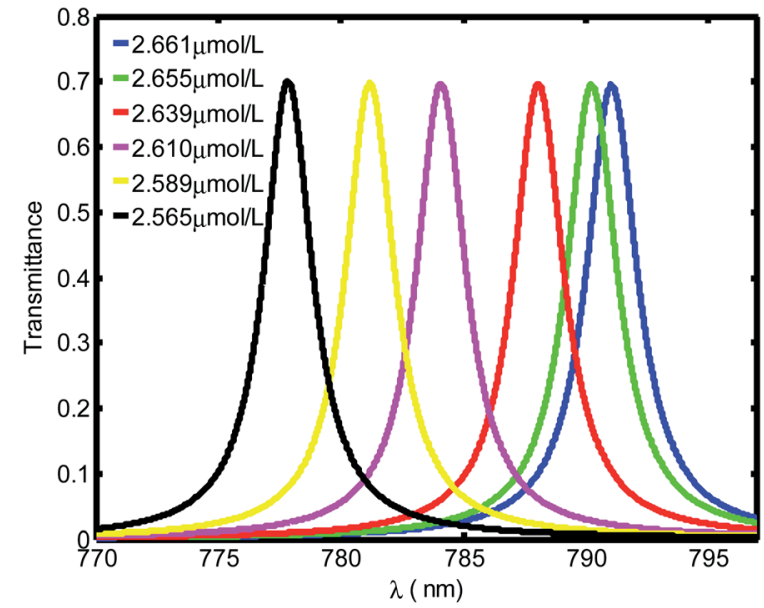

Fig. 3 Evolution of resonant peak of defective PC as the function of wavelength with different creatinine concentrations in blood, C. Here, $C$ is set between $2.565 \mu \mathrm{mol} \mathrm{L}-1$ and $2.661 \mu \mathrm{mol} \mathrm{L}-1$.

$$
T=\frac{P_{\mathrm{s}}}{P_{o}}\left|t^{2}\right|
$$

Finally, the sensitivity of the 1D-PCs can be defined as the ratio of the resonant peak wavelength to the contrast index using the following equation: ${ }^{\mathbf{4 4}}$

$$
S=\frac{\Delta \lambda}{\Delta n}
$$

with $\Delta \lambda=\lambda_{\mathrm{ab}}-\lambda_{\mathrm{n}}$ and $\Delta n=n_{\mathrm{ab}}-n_{\mathrm{n}}$ are the differences of wavelength and contrast index at normal and abnormal positions, respectively.

\section{Results and discussions}

The transmittance properties of the proposed biosensor based on the 1D defective PC are investigated. The effect of relative parameters related to creatinine defects such as blood creatinine concentration, thickness and incident angle are examined in order to achieve an efficient and stable biosensor.

In the numerical calculations, the considered material parameters of multilayered stacks of layer $\mathrm{A}\left(\mathrm{MgF}_{2}\right)$ and layer $\mathrm{B}$ $\left(\mathrm{CeO}_{2}\right)$ are the dielectrics of these materials wherein the thickness and refractive index were assumed to be $d_{\mathrm{A}}=d_{\mathrm{B}}=0.1 \mu \mathrm{m}$ and $n_{\mathrm{A}}=1.37$ and $n_{\mathrm{B}}=2.4$, respectively. Further, the thickness of blood creatinine sample considered was $230 \mathrm{~nm}$. The defect layer is considered in the blood creatinine sample under different concentrations.

Fig. 2 shows the transmittance spectra as a function of wavelengths through the defective 1D-PC at a blood creatinine concentration of $80.9 \mu \mathrm{mol} \mathrm{L}{ }^{-1}$. It is obvious that a resonant transmittance peak called defect mode within the enhancement
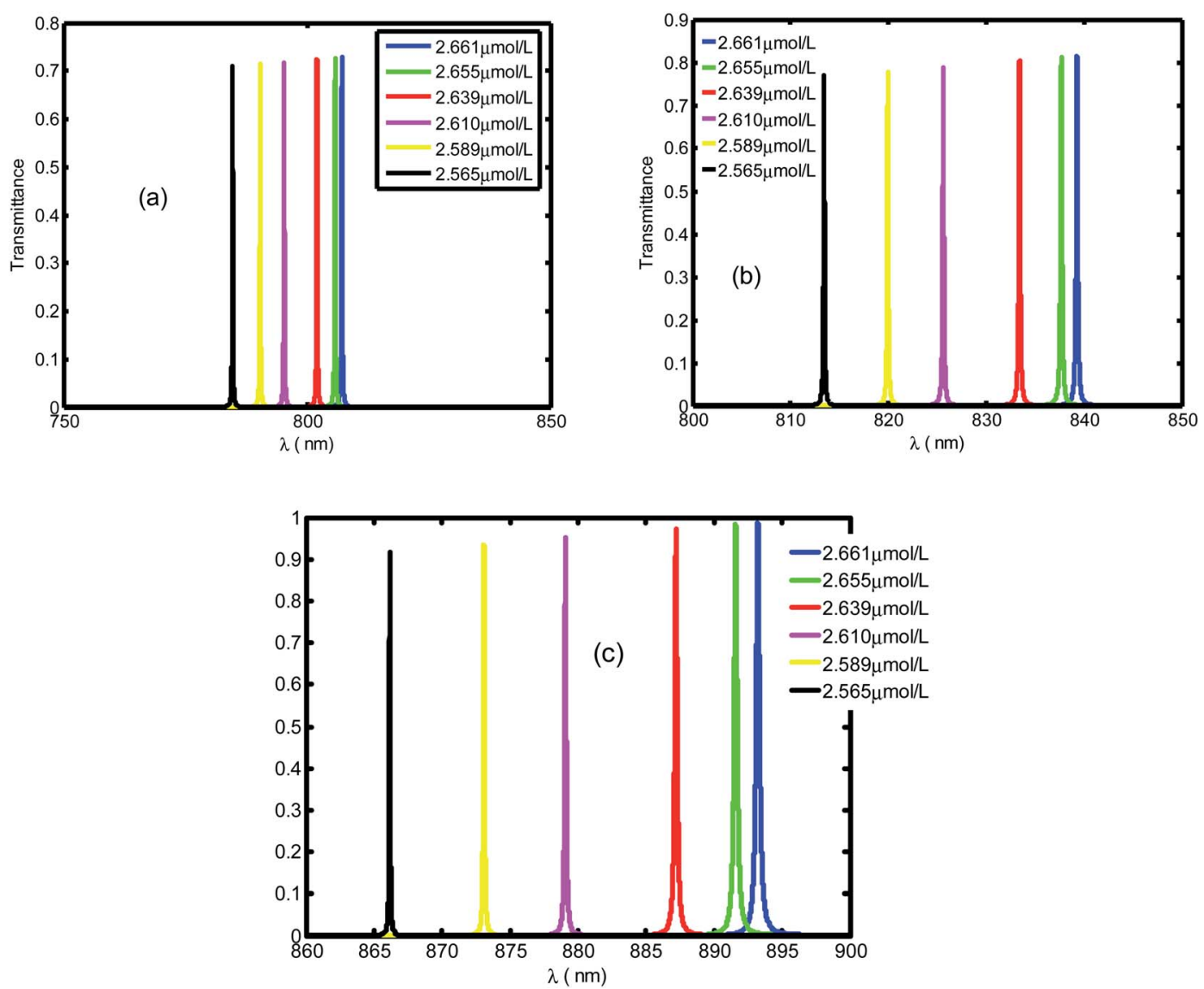

Fig. 4 The transmittance spectrum of defective PC as a function of wavelength with different values of creatinine concentration in blood, $d_{d}$. Here, the values of $d_{d}$ are set at $1 \mu \mathrm{m}$ (a), $2 \mu \mathrm{m}$ (b) and $3 \mu \mathrm{m}$ (c). 
of photonic band gap (PBG) for the seventh order of periodicity is observed. Further, the PBG prohibited the propagation of wave at a corresponding wavelength range [650-927.8 nm] and allowed only the wavelength of the defect mode.

Table 1 shows the evolution of creatinine concentration ( $\mu \mathrm{mol} \mathrm{L}^{-1}$ ) versus the refractive index. It is obvious that the creatinine concentration in the sample increases with the refractive index.

Fig. 3 shows the evolution of transmittance beam through the proposed biosensor based on defective PC at normal inci-

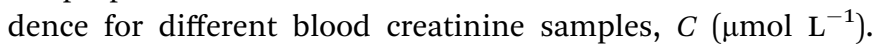
Here, $C$ is set to be between $2.565 \mu \mathrm{mol} \mathrm{L}^{-1}$ and $2.661 \mu \mathrm{mol} \mathrm{L}^{-1}$. By increasing the blood creatinine concentration, the defect mode gets shifted towards longer wavelengths. The aforementioned shift is due to the position dependent refractive index of the defect layer. Thus, this mobility is also interpreted by the condition of standing wave $\Delta=m \lambda=n_{\text {eff } G},{ }^{45}$ where $\Delta, m, n_{\text {eff }}$ and $G$ are the optical path difference, an integer, effective refractive index and geometric path difference, respectively. The proposed biosensor exhibited a sensitivity of about $136.4 \mathrm{~nm}$ per RIU at a normal incident angle.

Fig. 4 presents the emplacement of resonant peaks within the PBG at different regions of wavelengths. We noticed that the presented defect mode shift towards longer wavelengths when we increase the thickness of defect layer. We illustrated the enhanced amplitude and number of resonant peaks for higher value of thicknesses. Thus, we obtain high sensitivity with respect to the position of defect mode as we increase the thickness. This behavior is approved experimentally. ${ }^{46}$ By increasing $d_{\mathrm{d}}$, the geometrical path difference of defect peaks, the sensitivity rises from $136.4 \mathrm{~nm}$ per RIU at $d_{\mathrm{d}}=1 \mu \mathrm{m}$ to $286.5 \mathrm{~nm}$ per RIU at $d_{\mathrm{d}}=3 \mu \mathrm{m}$.

Fig. 5 displays the same behavior in Fig. 4 with resonant peaks and the defect mode shifted to longer wavelength when the defect layer thickness increased. In addition in Fig. 5 we illustrate an enhanced amplitude and number of resonant peaks for high value of thickness. Thus, we obtain an efficient sensitivity in which value increase with position of defect mode. This behavior is approved experimentally by work. ${ }^{46}$ By increasing $d_{\mathrm{d}}$ the geometrical path difference of defect peaks leads to rising the sensitivity from $136.4 \mathrm{~nm}$ per RIU at $d_{\mathrm{d}}=1$ $\mu \mathrm{m}$ to $286.5 \mathrm{~nm}$ per RIU at $d_{\mathrm{d}}=3 \mu \mathrm{m}$.

Fig. 6 shows the sensitivity (nm per RIU) of the proposed biosensor based on PC versus the thicknesses of defect layer, $d_{\mathrm{d}}$. We see that the sensitivity increases with $d_{\mathrm{d}}$ and follows the linear fitted equation: $S=137.62+0.057069 d_{\mathrm{d}}$. Here, $R=0.93967$ is the correlation coefficient between the linear fitting and the simulation data. $d_{\mathrm{d}}=3 \mu \mathrm{m}$ is set as the maximum thickness because an excess
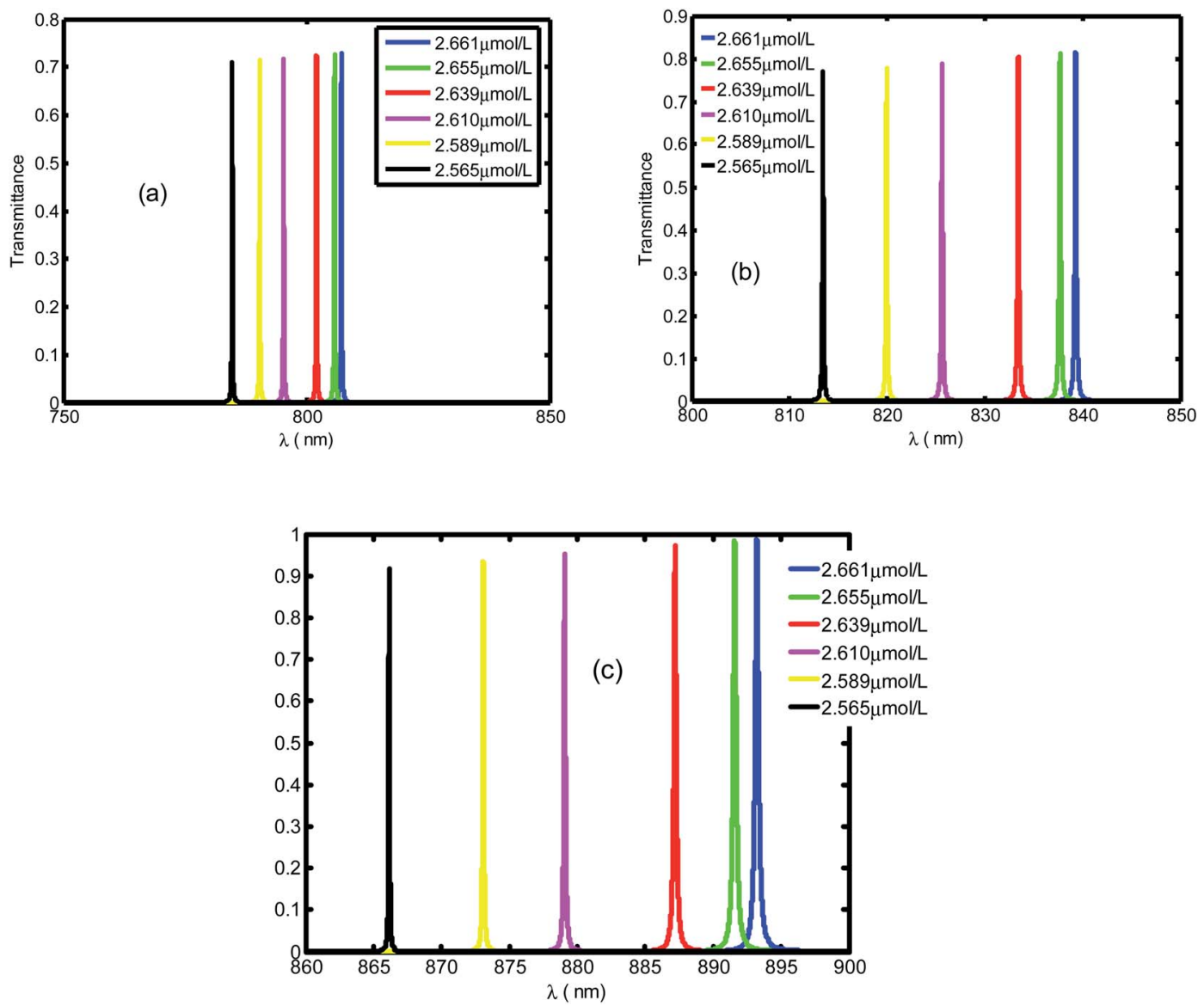

Fig. 5 The transmittance spectrum of defective PC as the function of wavelengths with different value of creatinine concentration in blood $d_{d}$. Here, $d_{d}$ is set at $1 \mu \mathrm{m}, 2 \mu \mathrm{m}$ and $3 \mu \mathrm{m}$, respectively. 


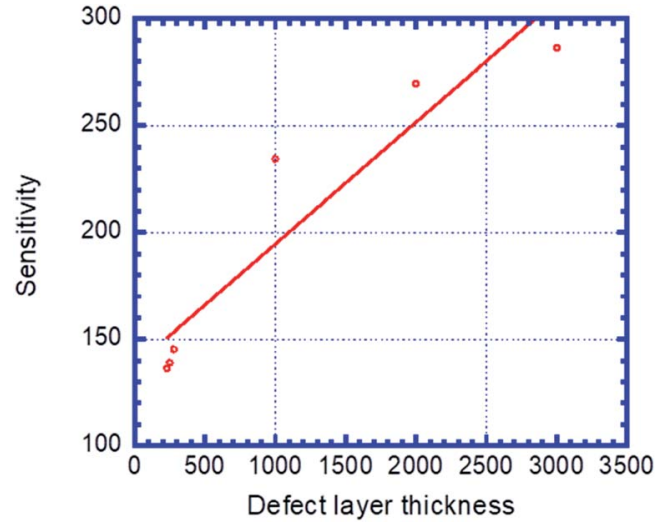

Fig. 6 Sensitivity of the designed biosensor based on defective PC versus the thickness of creatinine concentration sample, $d_{\mathrm{d}}$.

of this corresponding value causes the appearance of new peaks that disturb the performance of the sensor.

Further, we analyzed the dependence of resonant peaks on the incident angle. For the seventh order of periodicity, we plotted the transmittance spectra as a function of the wavelength for different incident angles. Here, the incident angles

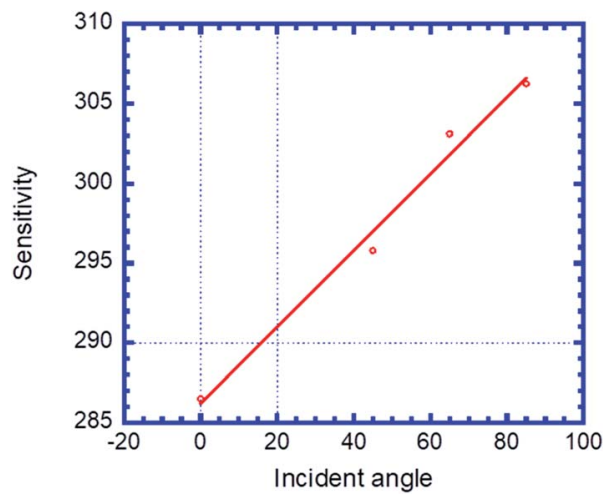

Fig. 8 Sensitivity of the proposed biosensor based on defective PC versus the incident angle for the considered creatinine blood concentration.

are set at $\theta=45^{\circ}$ (Fig. 7(a)), $\theta=65^{\circ}$ (Fig. 7(b)), and $\theta=85^{\circ}$ (Fig. 7(c)). It can be seen from the Fig. 7 that with increasing angle of incidence, the defect mode shifts down to lower wavelengths, while the amplitudes of insert resonant peaks increase and become sharper as we increase the incident angle. We can explain this behavior in Fig. 7 based on Bragg Snell's
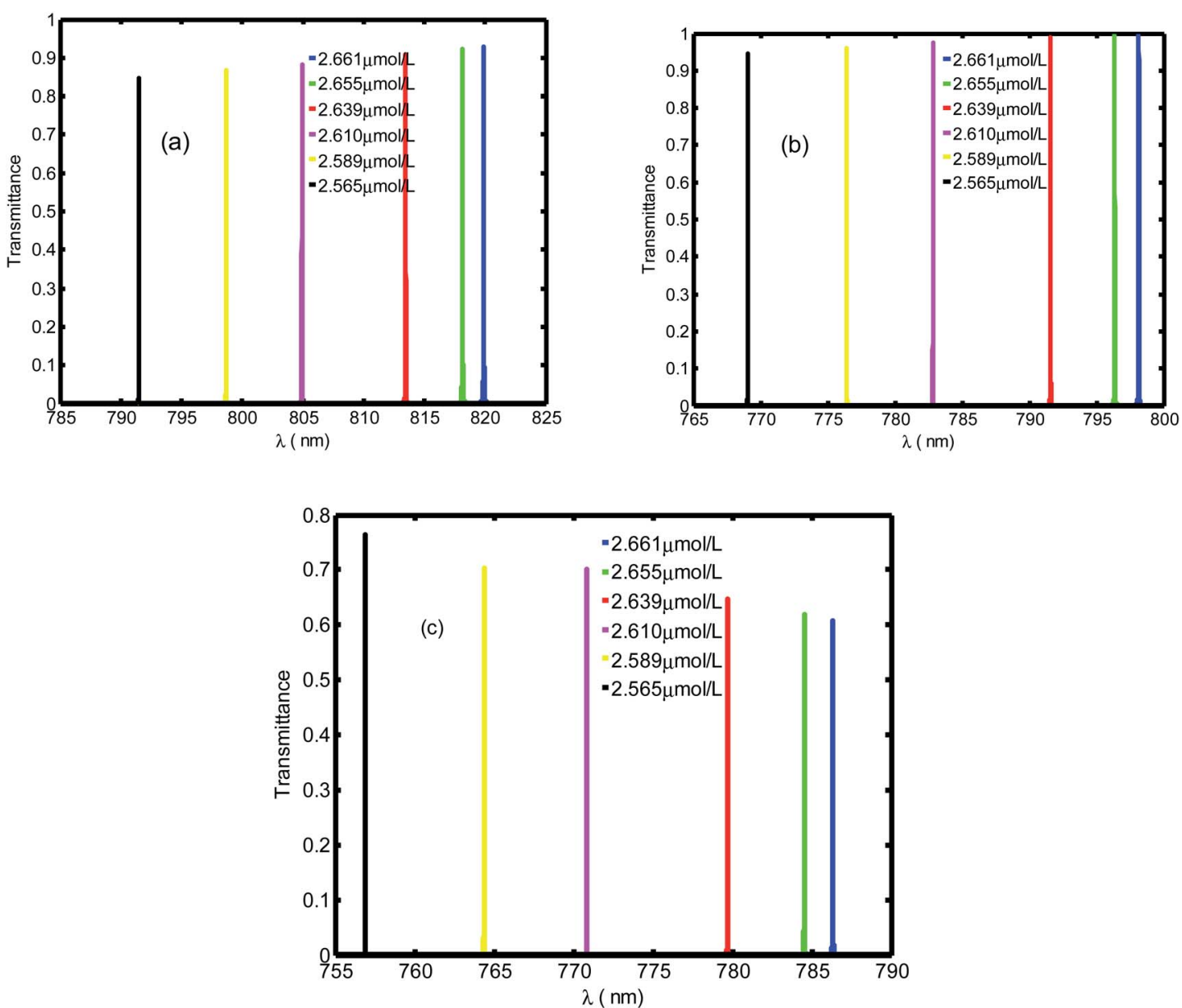

Fig. 7 Transmittance spectra of the proposed biosensor based on defective $\mathrm{PC}$ at different oblique incidences with $\theta$ are set at (a) $\theta=45^{\circ}$, (b) $\theta=$ $65^{\circ}$, and (c) $\theta=85^{\circ}$. 
Table 2 Properties of the proposed biosensor based on defective PC for different values of creatinine concentrations in blood $\left(\mu \mathrm{mol} \mathrm{L}^{-1}\right.$ )

\begin{tabular}{|c|c|c|c|c|c|c|}
\hline $\begin{array}{l}\text { Creatinine blood } \\
\text { sample concentration }\left(\mu \mathrm{mol} \mathrm{L}{ }^{-1}\right)\end{array}$ & $N$ & $S(\mathrm{~nm}$ per RIU) & $\lambda_{\mathrm{d}}(\mathrm{nm})$ & $\lambda_{\text {FWHM }}(\mathrm{nm})$ & $Q \times 10^{5}$ & $\mathrm{FOM} \times 10^{4}(\mathrm{RIU})$ \\
\hline 80.9 & 2.661 & - & 756.9 & 0.003 & 2.5 & - \\
\hline 81.43 & 2.655 & 306.25 & 764.4 & 0.003 & 2.5 & 10.3 \\
\hline 82.3 & 2.639 & 306.25 & 770.9 & 0.004 & 1.9 & 7.6 \\
\hline 83.3 & 2.610 & 306.25 & 779.7 & 0.0075 & 1.03 & 4.08 \\
\hline 84.07 & 2.589 & 306.25 & 784.5 & 0.01 & 0.78 & 3.06 \\
\hline 85.28 & 2.565 & 306.25 & 786.3 & 0.02 & 0.39 & 1.5 \\
\hline
\end{tabular}

law, ${ }^{47} \lambda=2 d \sqrt{n_{\mathrm{eff}}{ }^{2}-\sin ^{2} \theta}$, where $m, \lambda, d$ and $n_{\text {eff }}$ are the constructive diffraction order, the wavelength of the maximum reflected intensity, the period, and the effective refractive index, respectively.

Fig. 8 illustrates the variation in sensitivity (nm per RIU) of the proposed biosensor based on the defective PC with the angle of incidence. We see that the sensitivity ( $\mathrm{nm}$ per RIU) increases with the angle of incidence due to the increase in amplitude of the defect mode. The linear fitted plot of sensitivity versus incident angle follows the equation: $S=286.23+0.23966 \theta$ with $R=0.96947$, which is the correlation coefficient between the linear fitting and the simulation data.

The proposed biosensor designed (by alternating $\mathrm{MgF}_{2} / \mathrm{CeO}_{2}$ ) with creatinine sample defect exhibited an efficient sensitivity around $305 \mathrm{~nm}$ per RIU at high incident angles, which is not achieved for ordinary sensors, such as the theoretical optical sensor based on single nanobeam air-mode cavity (SNAC) with a sensitivity, $S=103 \mathrm{~nm}$ per RIU proposed by Yang et al. ${ }^{48}$ and the waveguide based sensor with a sensitivity, $S=282.4 \mathrm{~nm}$ per RIU proposed by Bagci and Akaoglu. ${ }^{49}$

To show the performance of the proposed biosensor based on the defective creatinine PC, we calculated the quality factor, $Q=\frac{\lambda_{\mathrm{d}}}{\lambda_{\mathrm{FWHM}}}$, and the ratio of sensitivity to full width at half maximum of the resonant peaks called the "figure of merit" FOM $=\frac{S}{\lambda_{\text {FWHM }}}$, where $\lambda_{\mathrm{d}}, \lambda_{\text {FWHM }}$ and $S$ are the resonant wavelength, the full width at half maximum (FWHM) of defect mode and sensitivity, respectively.

Table 2 shows the performance of our proposed biosensor that exhibits a high $Q$-factor about $2.5 \times 10^{5}$ at $80.9 \mu \mathrm{mol} \mathrm{L}^{-1}$ and a FOM about $1.03 \times 10^{5}$ (RIU) at $81.43 \mathrm{mmol} \mathrm{L}^{-1}$. Finally, we calculated the limit of detection (LOD) for the proposed sensor from the equation: $\mathrm{LOD}=\lambda /(20 \times S \times Q) \cdot{ }^{50,51}$ From the simulation results in Table 2 , LOD is equal $1.04 \times 10^{-6}$ RIU. The value of LOD is very low, which means that the proposed sensor is efficient as it is capable of resolving very small changes in the refractive index.

\section{Conclusion}

In this paper, a photonic biosensor based on 1D defective PC with creatinine blood sample has been developed. We theoretically investigated the performance of the proposed biosensor using the TMM approach. It is found that characteristics of the biosensor can be tuned by related parameters, such as concentration of creatinine in blood, thickness of defect layer and angle of incidence. From estimated parameters, we have obtained an efficient biosensor with high sensitivity approximating to $306.25 \mathrm{~nm}$ per RIU, which has not been achieved by ordinary optical sensors.

\section{Conflicts of interest}

The authors declare they have no conflicts of interest.

\section{Acknowledgements}

The authors are thankful to the Deanship of Scientific Research - Research Center at King Khalid University in Saudi Arabia for funding this research (code number: G.R.P-35-1441/2020).

\section{References}

1 E. Yablonovitch, Inhibited spontaneous emission in solidstate physics and electronics, Phys. Rev. Lett., 1987, 58, 2059.

2 E. Yablonovitch, Photonic band-gap structures, J. Opt. Soc. Am. B, 1993, 10, 283-295.

3 J.-B. Lee, Mechanically tunable photonic crystal structure, Appl. Phys. Lett., 2004, 85, 4845.

4 A. H. Aly and F. A. Sayed, THz cutoff frequency and multifunction $\mathrm{Ti}_{2} \mathrm{Ba}_{2} \mathrm{Ca}_{2} \mathrm{Cu}_{3} \mathrm{O}_{10} / \mathrm{GaAs}$ photonic band gap materials, Int. J. Mod. Phys. B, 2020, 34(10), 2050091.

5 Y. Trabelsi, N. B. Ali, A. H. Aly and M. Kanzari, Tunable high Tc superconducting photonic band gap resonators based on hybrid quasi-periodic multilayered stacks, Phys. C, 2020, 576, 1353706.

6 M. Haurylau, S. P. Anderson, K. L. Marshall and P. M. Fauchet, Electrical modulation of silicon-based twodimensional photonic band gap structures, Appl. Phys. Lett., 2006, 88, 1-3.

7 A. H. Aly, H. A. Elsayed, A. A. Ameen and S. H. Mohamed, Tunable properties of one-dimensional photonic crystals that incorporate a defect layer of a magnetized plasma, Int. J. Mod. Phys. B, 2017, 31, 1750239.

8 S. M. Shaban, A. Mehaney and A. H. Aly, Determination of 1propanol, ethanol, and methanol concentrations in water based on a one-dimensional phoxonic crystal sensor, Appl. Opt., 2020, 59(13), 3878-3885. 
9 B. Kim, Y. H. Kim, Y. Kim, J. Kang and W. Lee, An anion sensing photonic gel by hydrogen bonding of anions to the N-allyl-N0-ethyl urea receptor, J. Mater. Chem. A, 2014, 2, 5682 .

10 Y. Trabelsi, Output multichannel optical filter based on hybrid photonic quasicrystals containing a high-Tc superconductor, Photonic. Nanostruct., 2019, 36, 100724.

11 H. A. Arafa, S. E.-S. Abdel Ghany, B. M. Kamal and D. Vigneswaran, Theoretical studies of hybrid multifunctional $\mathrm{YaBa}_{2} \mathrm{Cu}_{3} \mathrm{O}_{7}$ photonic crystals within visible and infra-red regions, Ceram. Int., 2020, 46(1), 365369.

12 E. Desimoni and B. Brunetti, X-ray photoelectron spectroscopic characterization of chemically modified electrodes used as chemical sensors and biosensors: a review, Chemosensors, 2015, 3, 70-117.

$13 \mathrm{~K}$. Vijayashanthi and S. Robinson, Two-dimensional photonic crystal based sensor for pressure sensing, Photonic Sens., 2014, 4, 248-253.

14 N. R. Ramanujam, H. J. El-Khozondarb, V. Dhasarathan, S. A. Taya and A. H. Aly, Design of one dimensional defect based photonic crystal by composited superconducting material for bio sensing applications, Phys. B, 2019, 572, 42-55.

15 J. Kirsch, C. Siltanen, Q. Zhou, A. Revzin and A. Simonian, Biosensor technology: recent advances in threat agent detection and medicine, Chem. Soc. Rev., 2013, 42, 87338768.

16 S. Myung, P. T. Yin, C. Kim, J. Park, A. Solanki, P. I. Reyes, Y. Lu, K. S. Kim and K. B. Lee, Label-free polypeptide based enzyme detection using a graphene-nanoparticle hybrid sensor, Adv. Mater., 2012, 24, 6081-6087.

17 P. Sharma and P. Sharan, An analysis and design of photonic crystal based biochip for detection of glycosuria, IEEE Sensor. J., 2015, 15, 5569-5575.

18 A. H. Aly and Z. A. Zaky, Ultra-sensitive photonic crystal cancer cells sensor with a high-quality factor, Cryogenics, 2019, 104, 102991.

19 Y. Cui and C. M. Lieber, Nanowire nanosensors for highly sensitive and selective detection of biological and chemical species, Science, 2001, 293, 1289-1292.

20 M. Piliarik, M. Vala, I. Tichý and J. Homola, Compact and low-cost biosensor based on novel approach to spectroscopy of surface plasmons, Biosens. Bioelectron., 2009, 24, 3430-3435.

21 J. T. Heeres, S. H. Kim, B. J. Leslie, E. A. Lidstone, B. T. Cunningham and P. J. Hergenrother, Identifying modulators of protein-protein interactions using photonic crystal biosensors, J. Am. Chem. Soc., 2009, 131, 18202.

22 J. Hu, X. W. Zhao, Y. J. Zhao, J. Li and W. Y. Xu, Photonic crystal hydrogel beads used for multiplex biomolecular detection, J. Mater. Chem., 2009, 19, 5730.

23 H. Wang, K. Tong, C. Gu, W. Zeng and J. Lu, Two dimensional photonic crystal biosensors, Adv. Mater. Res., 2013, 683, 419.
24 P. Kvasnička, K. Chadt, M. Vala, M. Bocková and J. Homola, Toward single-molecule detection with sensors based on propagating surface plasmons, Opt. Lett., 2012, 37, 163-165.

25 S. V. Marchenko, T. A. Sergeyeva, A. L. Kukla, A. S. Pavlyuchenko, E. K. Krasyuk, A. P. Soldatkin and A. V. El'skaya, Application of creatinine-sensitive biosensor for hemodialysis control, Biosens. Bioelectron., 2012, 15, 466-469.

26 S. Robinson and N. Dhanlaksmi, Photonic crystal based biosensor for the detection of glucose concentration in urine, Photonic Sens., 2017, 7, 11-19.

27 M. Yunianto, A. N. Permata, D. Eka, D. Ariningrum, S. Wahyuningsih and M. Ahmad, Design of a Fiber Optic Biosensor for Cholesterol Detection in Human Blood, Mater. Sci. Eng., 2017, 176, 012014.

28 S. Poeggel, D. S. Tosi, D. B. Duraibabu, G. Leen, D. McGrath and E. Lewis, Optical fibre pressure sensors in medical applications, Sensors, 2015, 15, 17115.

29 V. R. Dharnidharka, C. Kwon and G. Stevens, Serum cystatin $\mathrm{C}$ is superior to serum creatinine as a marker of kidney function: a meta-analysis, Am. J. Kidney Dis., 2002, 40, 221226.

30 S. S. Waikar, R. A. Betensky and J. V. Bonventre, Creatinine as the gold standard for kidney injury biomarker studies, Nephrol., Dial., Transplant., 2009, 24, 3263.

31 M. Parvesh, P. Ohlsson and I. BjOrkhem, Combined enzymic- Jaffe method for determination of creatinine in serum, Clin. Chem., 1981, 27, 8-21.

$32 \mathrm{~W}$. D. Langley and M. Evans, The determination of creatinine with sodium 3,5-dinitrobenzoate, J. Biol. Chem., 1936, 115, 333-341.

33 I. Bjorkhem, R. Blomstrand and G. Ohman, Mass fragmentography of creatinine proposed as a reference method, Clin. Chem., 1977, 23, 2114-2121.

34 M. A. Awad and A. H. Aly, Experimental and theoretical studies of hybrid multifunctional $\mathrm{TiO}_{2} / \mathrm{TiN} / \mathrm{TiO}{ }_{2}$, Ceram. Int., 2015, 45, 19036-19043.

35 V. Revathy, C. Boopathi, K. Selvakumar, K. S. J. Wilson, S. A. Taya, A. H. Aly and M. S. M. Rajan, Nonlinear polarization in metal nanocomposite system based photonic crystals, Optik, 2019, 176, 78-84.

36 A. H. Aly, S. S. A. Ghany, M. Fadlallah, F. Salman and B. Kamal, Transmission and temperature sensing characteristics of a binary and ternary photonic band gap, J. Nanoelectron. Optoelectron., 2015, 10, 9-14.

37 N. Ayyanar, G. T. Raja, M. Sharma and D. S. Kumar, Photonic crystal fiber-based refractive index sensor for early detection of cancer, IEEE Sens. J., 2018, 18, 7093-7099.

38 J. Raveendran J, P. E. Resmi, T. Ramachandran, B. G. Nair and T. G. Satheesh Babu, Fabrication of a disposable nonenzymatic electrochemical creatinine sensor, Sens. Actuators, B, 2017, 243, 589-595.

39 C. Zeng, C. Luo, L. Hao and Y. Xie, The research on magnetic tunable characteristics of photonic crystal defect localized modes with a defect layer of nanoparticle, Chin. Opt. Lett., 2014, 12, 11602. 
40 W. Junge, B. Wilke, A. Halabi and G. Klein, Determination of reference intervals for serum creatinine, creatinine excretion and creatinine clearance with an enzymatic and modified Jaffé method, Clin. Chim. Acta, 2004, 344, 137-148.

41 T. Yao and K. Kotegawa, Simultaneous flow-injection assay of creatinine and creatine in serum by the combined use of a 16-way switching valve, some specific enzyme reactors and a highly selective hydrogen peroxide electrode, Anal. Chim. Acta, 2002, 462, 283-291.

42 A. C. Sharma, T. Jana, R. Kesavamoorthy, L. Shi, M. A. Virji, D. N. Finegold and S. A. Asher, A general photonic crystal sensing motif: creatinine in bodily fluids, J. Am. Chem. Soc., 2004, 126(9), 2973.

43 W. M. Nouman, S. E.-S. Abd El-Ghany, S. M. Sallam, A.-F. B. Dawood and H. A. Arafa, Biophotonic sensor for rapid detection of brain lesions using 1D photonic crystal, opt. Quantum Electron., 2020, 52(287), 1-14.

44 F. Bagci and B. Akaoglu, Enhancement of refractive index sensitivity in photonic crystal waveguide-based sensors by selective infiltration, Acta Phys. Pol., A, 2013, 124, 50.

45 A. H. Aly, Metallic and superconducting photonic crystal, J. Supercond. Novel Magn., 2008, 21, 421.
46 C. Zeng, C. Luo, L. Hao and Y. Xie, The research on magnetic tunable characteristics of photonic crystal defect localized modes with a defect layer of nanoparticle, Chin. Opt. Lett., 2014, 12, 11602.

47 E. Armstrong and C. O. Dwyer, Artificial opal photonic crystals and inverse opal structures-fundamentals and applications from optics to energy storage, J. Mater. Chem. C, 2015, 3, 6109.

48 D. Yang, H. Tian and Y. Ji, High-Q and high-sensitivity width-modulated photonic crystal single nanobeam airmode cavity for refractive index sensing, Appl. Opt., 2015, 54, 1-5.

49 F. Bagci and B. Akaoglu, Enhancement of refractive index sensitivity in photonic crystal waveguide-based sensors by selective infiltration, Acta Phys. Pol., A, 2013, 124, 50.

50 I. M. White and X. Fan, On the performance quantification of resonant refractive index sensors, Opt. Express, 2008, 16, 1020-1028.

51 M. El Beheiry, V. Liu, S. Fan and O. Levi, Sensitivity enhancement in photonic crystal slab biosensors, Opt. Express, 2010, 1822702-1822714. 